\title{
Spontaneous regression of a lumbar disk herniation
}

\author{
Michael J. Monument MD, Paul T. Salo MD
}
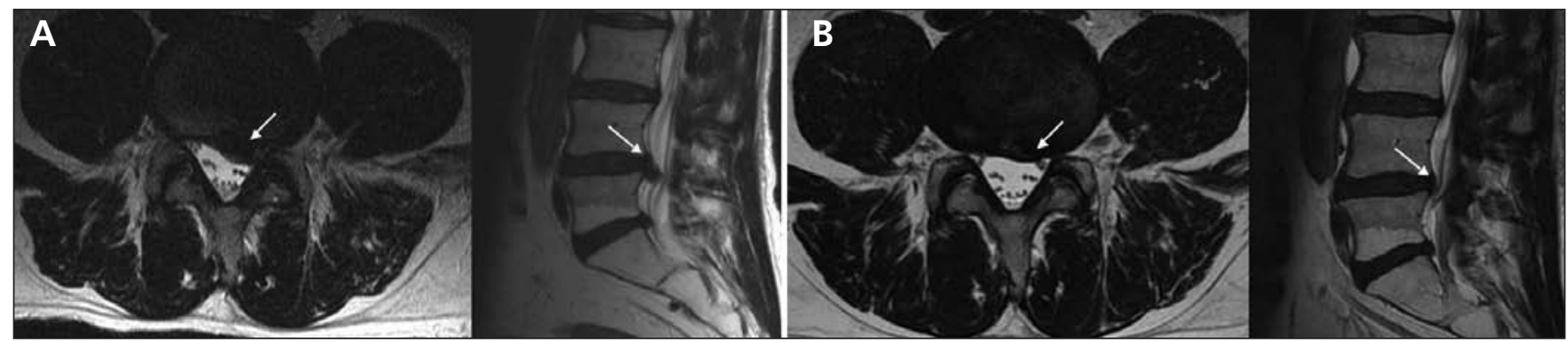

Figure 1: Axial and sagittal $T_{2}$-weighted magnetic resonance images of the lumbar spine of a 57-year-old man, (A) obtained shortly after an initial presentation for sciatica, showing a large paracentral L4-L5 disk herniation (arrows) compressing the traversing L5 nerve root, and (B) obtained six months later, showing significant resolution of the herniation (arrows).

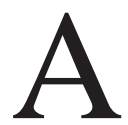

57-year-old man was referred by his family physician to our spine clinic for assessment of left-sided leg pain of six months' duration. His history was significant only for a spontaneous onset of lumbar back pain noted immediately after awkward bending six months prior, followed two to three days later by severe leg pain, paresthesias and ankle weakness, consistent with an L5 radiculopathy. His initial leg pain had been so intense that he had briefly required a wheelchair for ambulation, despite high-dosage oral narcotics. Magnetic resonance imaging performed shortly after the onset of pain had shown a large left-sided paracentral disk herniation at the L4-L5 disk space, compressing the traversing L5 nerve root, that was consistent with the patient's symptoms (Figure 1A). While awaiting referral to our spine clinic, he underwent activity modifications, physiotherapy and a tapering course of oral narcotics.

At the time of assessment at our clinic, six months after the onset of symptoms, the patient no longer had leg pain or physical findings of nerve root tension. He did have residual left-sided grade 4 out of 5 weakness of his great toe extensor and ankle dorsiflexors. At that time, a second magnetic resonance imaging study showed substantial resolution of the herniated L4-L5 disk (Figure 1B).

Sciatica due to a herniated lumbar intervertebral disk is common and has an estimated point prevalence of roughly $5 \%$ in the general adult population. ${ }^{1}$ Numerous prospective studies have reported excellent recovery of acute sciatica in the absence of significant, progressive neurologic deficits, unmanageable radicular pain or sphincter disturbance with nonoperative treatment, including mobilization, nonsteroidal anti-inflammatory drugs and physical therapy. ${ }^{2-4}$ The spontaneous disappearance of intervertebral disk herniation is well documented, yet the exact mechanism of this process remains unresolved. Proposed mechanisms include mechanical retraction of the herniated nucleus, gradual dehydration and shrinkage of the disk, or enzymatic degradation of herniated disk material. ${ }^{3-6}$ Regardless, the resolution of acute sciatica can be expected in greater than $50 \%$ of patients receiving nonoperative treatment, ${ }^{2-4}$ supporting the rationale for nonoperative management in most patients.

\section{References}

1. Heliövaara M, Makela M, Knekt P, et al. Determinants of sciatica and low-back pain. Spine (Phila Pa 1976) 1991, 16:608-14

2. Weinstein JN, Tosteson TD, Lurie JD, et al. Surgical vs nonoperative treatment for lumbar disk herniation. The Spine Patient Outcomes Research Trial (sport): a randomized trial. JAMA 2006;296:2441-50.

3. Chou R, Loeser JD, Owens DK et al; for the American Pain Society Low Back Pain Guideline Panel. Interventional therapies, surgery, and interdisciplinary rehabilitation for low back pain: an evidence-based clinical practice guideline from the American Pain Society. Spine 2009;34:1066-77.

4. Chou R, Qaseem A, Snow V, et al.; for the Clinical Efficacy Assessment Subcommittee of the American College of Physicians and the American Pain Society Low Back Pain Guidelines Panel. Diagnosis and treatment of low back pain: a joint clinical practice guideline from the American College of Physicians and the American Pain Society. Ann Intern Med 2007;147:478-91.

5. Komori H, Shinomiya K, Nakai O, et al. The natural history of herniated nucleus pulposus with radiculopathy. Spine (Phila Pa 1976) 1996;21:225-9.

6. Fager CA. Observations on spontaneous recovery from intervertebral disc herniation. Surg Neurol 1994;42:282-6.
Competing interests: None declared.

This article has been peer reviewed.

Affiliations: From the McCaig Institute for Bone and Joint Health, and the Division of Orthopaedic Surgery, Faculty of Medicine, University of Calgary, Calgary, Alta.

Correspondence to: Dr. Michael Monument, mjmonume@ucalgary.ca

CMAJ 2011. DOI:10.1503 /cmaj.091918 